\title{
COMUNICACIONES
}

\section{Búsqueda de huevos de anquilostomideos y toxocarídeos en el suelo de residencias y escuelas en el barrio de dois irmãos, Recife-PE (Brasil)}

\author{
ANA MARÍA ALVES LIMA*, LEUCIO CÂMARA ALVES*, MARÍA APARECIDA DA GLORIA \\ FAUSTINO*, NADJA MARÍA SILVA DE LIRA*, ÂNGELA MAGALHÃES**, MARILENE MARÍA DE \\ LIMA*, WHAUBTYFRAN CABRAL TEIXEIRA*, JOÃO CARLOS GOMES BORGES*, e \\ DANILLO DE SOUZA PIMENTEL*
}

\section{CONTAMINATION OF SOIL WITH Ancylostoma spp and Toxocara spp EGGS IN DOIS IRMAOS AREA, CITY OF RECIFE}

This study was carried out to investigate the contamination of soil garden, back yard from 149 houses and two public schools playgrounds areas with Ancylostoma spp and Toxocara spp eggs from the Córrego da Fortuna community, Dois Irmãos area in City of Recife-PE, Brazil. The samples were collected during summer and winter and analyzed by centrifugal flotation technique by using of 33\% Zinc sulfate solution. The results showed Ancylostoma spp. and Toxocara spp eggs in 4.69\% (07/ $149)$ and $8.73 \%$ (13/149) of samples respectively from garden, back yard, but eggs of nematodes were not recovered from public schools playgrounds. The study indicates that exposure of human to helminthes with zoonotic potential may be higher indoor than outdoor.

Key words: Ovos de Ancylostoma spp., ovos de Toxocara spp., solo.

\section{INTRODUCCIÓN}

El suelo puede constituir una vía de transmisión para varias zoonosis parasitarias ${ }^{1}$, entre las cuales destaca la Larva Migrans Visceral (LMV) y Larva Migrans Cutánea (LMC), causada principalmente por la migración de larvas de Toxocara sp y larvas de Ancylostoma $\mathrm{sp}^{1}$.

El creciente número de perros y gatos domésticos y errantes, asociado al fácil acceso de estos animales a las zonas de recreo, dentro y fuera de los domicilios, aumenta el riesgo de infección, principalmente para los niños ${ }^{2}$.

En zonas urbanas, la contaminación ocasionada por materia fecal de perros, está directamente relacionada con los hábitos culturales de la población, que favorecen la dispersión de heces en lugares públicos ${ }^{3}$. El papel de perros y gatos como huéspedes definitivos de varias zoonosis, ha sido ampliamente estudiado y reconocido como un problema importante de salud pública.

El objetivo de este trabajo fue investigar la

* Laboratorio de Enfermedades Parasitarias de la Universidad Federal Rural de Pernambuco (Brasil).

** Medica Veterinaria en la Ciudad de Recife - PE (Brasil).

Correspondencia: Prof Dr. Leucio Câmara Alves. Laboratório de Doenças Parasitárias dos Animais Domésticos. Departamento de Medicina Veterinária da Universidade Federal Rural de Pernambuco. Av. Dom Manuel de Medeiros, S/N, Dois Irmãos, Recife- PE, Brasil CEP.: 52171900-(81) 33206422/ (81) 33206423.-(81) 32698193. 
presencia de huevos de anquilostomatídeos y toxocarídeos en el suelo de residencias y de las áreas de recreo de escuelas situadas en el Bairro de Dois Irmãos, en Recife- PE.

\section{MATERIAL Y MÉTODOS}

Se eligió la comunidad de Córrego da Fortuna (34 $57^{\circ}$ '06" Sur de Latitude Meridional y a $08^{\circ}$ 00' 29" Oeste de longitud $)^{4}$, donde fueron investigados los suelos de residencias y escuelas públicas y particulares ubicados en la comunidad.

La comunidad está situada en el Barrio de Dois Irmãos, pertenciente a la Región PolíticoAdministrativa 03 (RPA 3) de la ciudad de Recife, PE en BRASIL. Con cerca de 6.000 habitantes, la comunidad está dividida en ocho microáreas, siendo una de las áreas con remanentes de la selva atlántica donde las características ambientales están también fuertemente relacionadas a la existencia de áreas verdes, en parcelas privadas, básicamente compuestas por pomares e canteros de plantas ornamentales ${ }^{5}$. El suelo posee características que predominan entre arcilloso y arenoso ${ }^{6}$. Como en toda a región metropolitana de Recife, el clima puede es caliente y húmedo, con precipitaciones más intensas entre Abril y Julio?.

Se recogieron muestras de suelo de jardines, patios, áreas de construcción de las residencias visitadas, de las cajas de arena y suelo de las áreas de recreo de las escuelas investigadas durante la estación seca y lluviosa.

La recogida de las muestras fue realizada con la ayuda de una pala de jardínero, en dos áreas diferentes de cada local, con una distancia mínima de cinco metros de cualquier material fecal. Fueron retirados aproximadamente 250 gramos de suelo, de una profundidad de hasta ocho centímetros, guardados en bolsas de plástico, identificadas y conservadas refrigeradas a $4^{\circ} \mathrm{C}$ hasta su estudio en el laboratorio ${ }^{8}$.

Las muestras se llevaron al Laboratorio de Enfermedades Parasitarias de la Universidad Federal Rural de Pernambuco (Brasil), donde fueron lavadas con $50 \mathrm{ml}$ de agua potable y tamizadas.

Del material resultante, $15 \mathrm{ml}$ fueron colocados en un tubo cónico de centrífuga y centrifugados a $1.000 \mathrm{G}$ durante cinco minutos. Posteriormente, se descartaba el sobrenadante y se procesaba nuevamente por la técnica de flotación con sulfato de zinc al 33\%. Después de la centrifugación, los tubos eran colocados en gradillas para tubos de ensayo, cubiertos con cubreobjetos (15 $\mathrm{mm} \times 5 \mathrm{~mm})$, durante 15 minutos. Posteriormente, los cubreobjetos eran colocados sobre láminas para microscopía (26 x $76 \mathrm{~mm}$ ) y llevadas al microscopio óptica para su observación $(100 \mathrm{x})$, con dos repeticiones para cada muestra 9 .

El análisis estadístico se realizó por chicuadrado $\left(\chi^{2}\right)^{10}$, utilizando el Programa Word Excel 7.0 para verificar la asociación entre las variables tipo de suelo y estación seca o lluviosa y la positividad de las muestras de suelo.

\section{RESULTADOS Y DISCUSIÓN}

De las 149 muestras de suelo residenciales analizadas, el 8,73\% (13/149) fueron positivas para huevos de toxocarídeos (Figura 1) y el $4,69 \%(07 / 149)$ fueron positivas para huevos de anquilostomatídeos (Figura 2).

Esos resultados son inferiores a los estudiados en plazas de la ciudad de Campo Grande (MS) en que fueron observados $10,8 \%$ de muestras positivas para el genero Toxocara $s p$ y $56,8 \%$ para el género Ancylostoma $\mathrm{sp}^{11}$.

Merece la pena destacar que la mayoría de los estudios realizados son relativos a la detección de un género de geohelminto. Los resultados obtenidos son inferiores a los descritos en el suelo de 17 parques ubicados en el área urbana de Lima, en Perú onde fueron encontrados un $70,6 \%$ de huevos de Toxocara $\mathrm{sp}^{12}$ y a los observados en áreas urbanas en la ciudad de Basrah, en Irak con una tasa de prevalencia del $17,3 \%$ de suelos contaminados por huevos de Toxocara $\mathrm{sp}^{13}$.

De la misma forma, la presencia de huevos de Ancylostoma sp encontrada fue inferior al 93,3\% observado en muestras de suelo recogidas de plazas públicas de la ciudad de Santa María $(\mathrm{RS})^{14}$ y al $22,2 \%$ encontrado en el suelo de escuelas y guarderías ${ }^{15}$, y al 76,3\% encontrado en la ciudad de Espírito Santo dos Pinhais (SP) ${ }^{2}$.

Sin embargo, los resultados obtenidos en relación a Toxocara fueron superiores a los observados en el suelo de 13 escuelas en la ciudad de Espírito Santo dos Pinhais (SP),en donde se obtuvo una frecuencia de apenas un $1,5 \%$. 


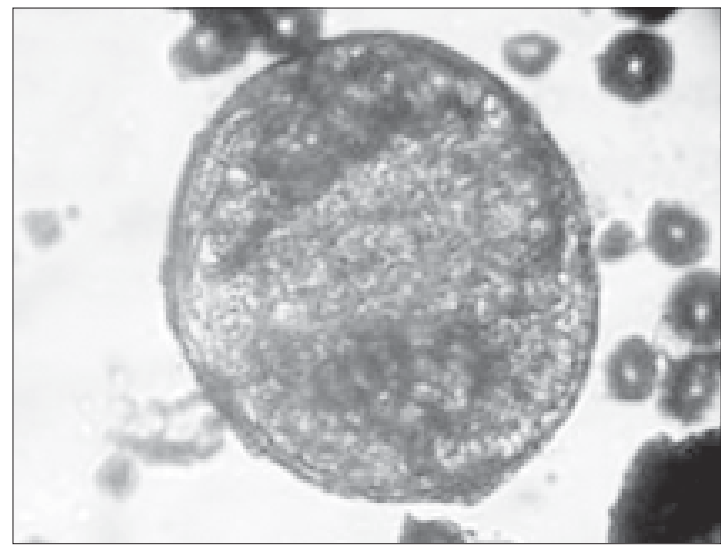

Figura 1. Huevo de toxocarídeo en muestra de suelo.

En ese trabajo, de las muestras positivas para huevos de anquilostomatídeos, todas provenían de suelo arenoso, mientras que, de las muestras positivas para huevos de toxocarídeos, el $69,23 \%$ (09/13) y el $30,77 \%$ (4/13) eran provenientes de suelo arenoso y arcilloso respectivamente.

Durante la estación seca, el 40\% (2/5) de las muestras de suelo residencial fue positivo para huevos de anquilostomatídeos en suelo de tipo arenoso y el $60 \%(3 / 5)$ de las muestras fue positivo para toxocarídeos en suelo de tipo arenoso. Del suelo residencial recogido en la estación lluviosa, el 33,33\% (5/15) de las muestras fueron positivas para huevos de anquilostomatideos en suelo arenoso, el 40\% (6/15) fueron positivas para huevos de toxocarideos en suelo arenoso y el 26,66\% (4/15) de las muestras fueron positivas para huevos de toxocarídeos en

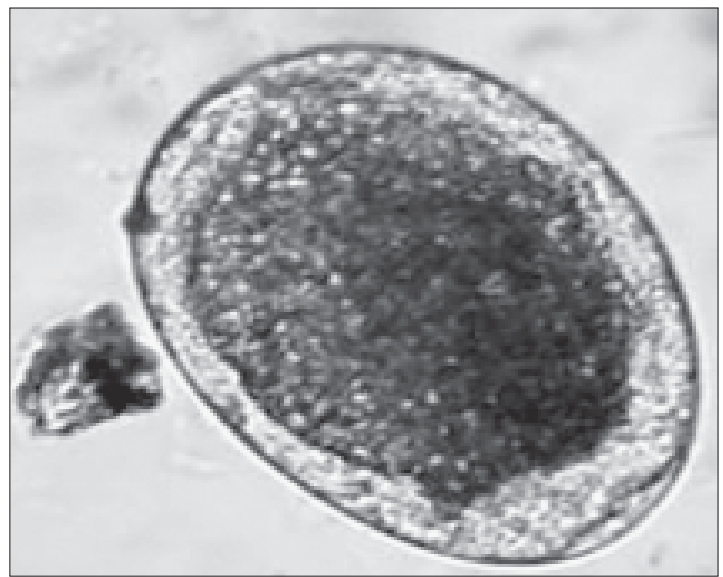

Figura 2. Huevo de anquilostomatídeo en muestra de suelo.

suelo arcilloso.

Los suelos arenosos favorecen la supervivencia de algunos agentes patógenos, particularmente aquellos pertenecientes a la familia Ancylostomatidae y dificultan la supervivencia de las bacterias. Por otro lado, el tiempo de supervivencia de esos patógenos aumenta en suelos húmedos ${ }^{6}$.

En un ambiente con una temperatura entre $15^{\circ} \mathrm{C}$ y $35^{\circ} \mathrm{C}$, la mayoría de los huevos de Toxocara se vuelven infectantes en un período de dos a cinco semanas, mientras que por debajo de esas condiciones ambientales, esos huevos requieren menos de una semana y temperaturas superiores a $35{ }^{\circ} \mathrm{C}$ provocan una rápida desintegración de los huevos ${ }^{16}$.

En un trabajo realizado en una localidad situada en la Región Metropolitana de Recife, se recogieron 25 muestras de suelo para detección

Tabla 1. Frecuencia absoluta y relativa de huevos de anquilostomatídeos y toxocarídeos en el suelo arenoso y arcilloso durante la estación seca y lluviosa, en residencias situadas en el barrio de Dois Irmãos, Recife - PE

\begin{tabular}{|c|c|c|c|c|c|c|c|c|c|}
\hline \multirow[t]{3}{*}{ Helminto } & \multicolumn{4}{|c|}{ Estación seca } & \multicolumn{4}{|c|}{ Estación lluviosa } & \multirow{3}{*}{ total } \\
\hline & \multicolumn{2}{|c|}{ Suelo arenoso } & \multicolumn{2}{|c|}{ Suelo arcilloso } & \multirow{2}{*}{$\begin{array}{l}\text { Suelo } \\
\text { F A }\end{array}$} & \multirow{2}{*}{$\begin{array}{c}\text { arenoso } \\
\text { F R }\end{array}$} & \multicolumn{2}{|c|}{ Suelo arcilloso } & \\
\hline & $\mathbf{F A}$ & F R & FA & F R & & & F A & F R & \\
\hline Anquilostomatideo & 02 & $40 \%$ & 00 & $0,0 \%$ & 05 & $3,3 \%$ & 00 & $0,0 \%$ & 07 \\
\hline Toxocarídeo & 03 & $60 \%$ & 00 & $0,0 \%$ & 06 & $40 \%$ & 04 & $26,6 \%$ & 13 \\
\hline Total & 05 & $25 \%$ & 00 & $0,0 \%$ & 11 & $55 \%$ & 04 & $20,0 \%$ & $\begin{array}{r}20 \\
100 \%\end{array}$ \\
\hline
\end{tabular}

F A = Frequencia absoluta; F R = Frequencia relativa; Estación x presencia helminto; Tipo de suelo x presencia helminto; $\mathrm{P}=0,087>0,05 ; \mathrm{P}=0,093>0,05$. 
de huevos de Toxocara y tres cuadrantes se encontraron contaminados siendo en dos de ellos el tipo de suelo predominante arcilloso. Dos muestras positivas para huevos de la familia Ascarididae procedían también de suelo tipo arcilloso9.

En un estudio realizado en Santiago de Chile, se recogieron muestras de suelo procedentes de 110 plazas y se encontró un $18,2 \%$ de positividad para huevos de Toxocara, de las cuales el 7,1\% provenía de suelo arenoso y el 16,7\% de suelo arcilloso, con el $16,7 \%$ recogidas durante el invierno el $8,9 \%$ en verano ${ }^{17}$.

En el análisis estadístico no se encontró asociación significativa entre las variables tipo de suelo, estación seca o lluviosa y la positividad de las muestras $(\mathrm{p}>0,05)$ (Tabla 1).

En la investigación coproparasitológica realizada en perros domiciliados de la comunidad estudiada, se observó que en 81 domicilios estudiados, el $65,4 \%$ eran positivos para huevos de helmintos, de los cuales el $83,1 \%$ fue positivo para Ancylostoma sp y el $11,3 \%$ positivo para Toxocara spp, donde el 83,1\% de los perros positivos no había recibido tratamiento antihelmíntico ${ }^{18}$.

Hay que destacar que de los 20 domicilios que tuvieron muestras positivas para huevos de anquilostomatídeos y toxocarídeos, en 19 había presencia de perros y/o gatos, en el $21,1 \%$ (4/ 19) había perros positivos para huevos de Ancylostoma sp y en el 10,52\% había perros positivos para huevos de Ancylostoma sp y Toxocara spp concomitantemente.

Por tanto, es importante resaltar que la presencia de perros parasitados domiciliados, sin la administración de anti-helmínticos, constituye un factor importante de riesgo para la contaminación del suelo con los desechos de esos animales, siendo un importante medio de diseminación de agentes causantes de zoonosis ${ }^{2}$.

Las muestras provenientes de las dos escuelas fueron negativas.

Con relación al resultado negativo de las muestras provenientes de las escuelas, de acuerdo con la información de las coordinaciones de esos establecimientos, la arena utilizada en las áreas de recreo es cambiada semestralmente y el acceso a esos locales de perros y gatos errantes está restringido, lo que supone un factor de importancia extrema para evitar la contaminación de esas áreas, pues, para el control de las parasitosis transmitidas por el suelo, como la LMC, en escuelas, no sirve únicamente el simple cambio de la arena de las áreas de recreo; cubrir las cajas de recreo y el examen del suelo cada vez que se realice el cambio, junto con las medidas que impidan el acceso de perros y gatos a esas áreas, son recomendables ${ }^{19}$.

\section{RESUMEN}

El objetivo de este artículo fue la investigación de la contaminación por huevos de Ancylostoma spp y Toxocara spp en el suelo del patio o jardín de 149 residencias y del área de recreo de dos escuelas situadas en la comunidad de Córrego da Fortuna no Bairro de Dois Irmãos, en Recife-PE (BRASIL). Las muestras fueron recogidas durante el invierno y verano y procesadas mediante la técnica de centrífugo-fluctuación, utilizando solución de sulfato de zinc al 33\%. De las muestras de suelo de residencias, 4,69\% (07/ 149) fueron positivas para huevos de Ancylostoma spp y 8,73\% (13/149) para huevos de Toxocara spp. Las muestras provenientes de las dos escuelas fueron negativas para huevos de nematodos. Los hallazgos muestran que la exposición de las personas por helmintos con potencial zoonótico, se demostró mayor en el ambiente domiciliar de que fuera deste ambiente.

\section{REFERENCIAS}

1.- NUNES C M, et al. Ocorrência de larva migrans na areia de áreas de lazer das escolas municipais de ensino infantil, Araçatuba, SP, Brasil. Rev Saúde Púb, São Paulo 2000; 34: 656-8.

2.- SANTOS R S, BONATO L C, MARQUES M P A. Ocorrência de agentes causais de larva migrans em areias destinadas à recreação em escolas de ensino infantil em Espírito Santo do Pinhal, SP, Brasil. Rev Ecossistema, Espírito Santo do Pinhal 2003; 28: 57-9.

3.- MILANO A M F, OSCHEROV E B. Contaminación por parasitos caninos de importancia zoonótica em playas de la ciudad de Corrientes, Argentina. Parasitol Latinoam 2002; 57: 3-4.

4.- PREFEITURA DO RECIFE. Secretaria de Saneamento. Prancha 01/02 (Sistema de Abastecimento de Água do Córrego da Fortuna). Janeiro de 2002.

5.- PREFEITURA DO RECIFE. Secretaria de Planejamento. Plano Diretor do Recife: Diagnóstico. II- A Dinâmica Físico-Territorial. 2006.

6.- FONSECA S P P. Tratamento de esgoto por deposição no solo. Fundação Estadual do Meio Ambiente - FEAM, Minas Gerais. Maio de 2005. 
7.- CPRH/GTZ. Qualidade do ar na Região Metropolitana do Recife - 1998. Governo do Estado de Pernambuco, Secretaria de Ciência, Tecnologia e Meio ambiente, Companhia Pernambucana de Meio ambiente (CPRH), Deustsche Gesellschaft für Technische Zuzammenarbeit (GTZ) 1999; 110 p.

8.- SANTARÉM V A, SARTOR I F, BERGAMO F M M. Contaminação, por ovos de Toxocara spp, de parques e praças públicas de Botucatu, São Paulo, Brasil. Rev Soc Brasil Med Trop 1998; 31: 6.

9.- LIMA J L, et al. Contaminação por ovos de Toxocara sp em solo no município de Moreno, Estado de Pernambuco, Brasil. Braz J Vet Res Animal Sci 2005; 42: 339-46.

10.- CALLEGARI-JACQUES S M. Bioestatística: princípios e aplicações. Porto Alegre: Artmed Editora, 2003; 255 p.

11.- ARAÚJO F R, et al. Contaminação de praças públicas de Campo Grande, Mato Grosso do Sul, Brasil, por ovos de Toxocara e Ancylostoma em fezes de cães. Rev Soc Brasil Med Trop 1999; 32: 125-30.

12.- CASTILLO Y, et al. Estudio epidemiológico de Toxocara canis en parques recreacionales del distrito de San Juan de lurigancho, Lima - Perú. Parasitol al Día 2001; 25: 301-9.

13.- MAHDI N K, ALI H A. Toxocara eggs in the soil of public places and schools in Basrah, Iraq. Ann Trop
Med Parasitol; 1993; 87: 201-5.

14.- CORRÊA G L B, MOREIRA W S. Contaminação do solo por ovos de Ancylostoma spp em praças públicas na cidade de Santa María, RS, Brasil. Rev Fac Zoot Vet e Agron 1995/1996; 213: 15-7.

15.- GUIMARÃES A M, et al. Ovos de Toxocara sp e larvas de Ancylostoma sp em praça pública de Lavras, MG. Rev Saúde Púb 2005; 39: 293-5.

16.- SCHANTZP M, GLICKMAN L T. Ascaridos de perros y gatos: un problema de salud publica y de medicina veterinaria. Bol Of San Panam, Washington, US, 1983.; 4: $571-85$

17.- SALINAS P, MATAMALA M, SCHENONE $\mathrm{H}$. Prevalencia de hallazgo de huevos de Toxocara canis em plazas de la Región Metripolitana de la ciudad de Santiago, Chile. Bol Chil Parasitol 2001; 57: 62.

18.- LIMA A M A, et al. Freqüência de enteroparasitos em cães domiciliados da comunidade do Córrego da Fortuna no bairro de Dois Irmãos na cidade do Recife, PE. In: JORNADA DE ENSINO, PESQUISA E EXTENSÃO, 6; SIMPÓSIO DE PÓS-GRADUAÇÃO, 7, 2006. Recife. Anais. Recife: Universidade Federal Rural de Pernambuco, 2006.

19.- ARAÚJO F R, et al. Larva migrans cutânea em crianças de uma escola em área do Centro-Oeste do Brasil. Rev Saúde Púb 2000; 34: 103-6.

\footnotetext{
Correspondencia a:

e-mail: leucioalves@gmail.com

amarial@walla.com
} 